\title{
Association between mental health and physical activity levels in people with Parkinson's disease during the COVID-19 pandemic: an observational cross-sectional survey in Brazil
}

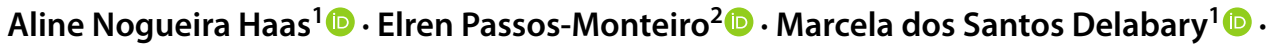

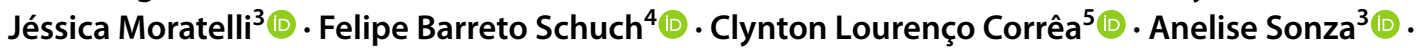 \\ Adriana Coutinho de Azevedo Guimarães ${ }^{3}$ (1) - Leonardo Alexandre Peyré-Tartaruga ${ }^{1}$ (1)
}

Received: 11 June 2021 / Accepted: 2 November 2021 / Published online: 14 January 2022

(c) The Author(s), under exclusive licence to Springer-Verlag Italia S.r.l., part of Springer Nature 2021

\begin{abstract}
Aims (1) To evaluate mental health symptoms in people with Parkinson's (PwP) in self-isolation, before and during the COVID-19 pandemic, in Brazil; (2) to explore associations between mental health and physical activity levels.

Methods An observational cross-sectional survey using retrospective data. PwP from the Brazilian territory, both sexes, no age limit, in self-isolation due to COVID-19 pandemic, were invited to complete an online self-administered and validated questionnaire. Demographic data (sex, age, Brazilian state they lived in, levels of education, and household income), days in self-isolation, time of diagnosis, and symptoms that bothered most were reported. Self-reported levels of physical activity and mental health symptoms (depression, anxiety, fear, and thoughts of death), before and during the COVID-19 pandemic, were assessed.

Results The participants were 156 individuals with PD (64 \pm 11 years), from both sexes (50\% women; 50\% men), resident in the 5 Brazilian regions. There was a worse on mental health symptoms over the time: anxiety [effect size $=-0.52 ; 95 \%$ CI $(-0.70 ;-0.28) ; p<.001$ ], fear [effect size $=-0.58 ; 95 \%$ CI $(-0.76 ;-0.34) ; p<.001$ ], and thoughts of death [effect size $=-0.43 ; 95 \%$ CI $(-072 ;-0.02) ; p=.001]$. A lower physical activity level during the pandemic is related to increased probability of thoughts of death [crude $\mathrm{OR}=1.84 ; 95 \% \mathrm{CI}(0.98 ; 3.46) ; p=0.05$; adjusted $\mathrm{OR}=2.98 ; 95 \% \mathrm{CI}(-0.01 ; 2.19)$; $p=0.05]$. Anxiety, fear and depression were not associated with physical activity levels.

Conclusions Anxiety, fear and thoughts of death worsened during the COVID-19 pandemic in comparison with the period before COVID-19. Lower physical activity level during the pandemic was related to an increased probability of thoughts of death.
\end{abstract}

Keywords COVID-19 $\cdot$ Parkinson's disease $\cdot$ Mental health $\cdot$ Physical activity

Marcela dos Santos Delabary

marcela_delabary@yahoo.com.br

1 Department of Physical Education, Federal University of Rio Grande do Sul, Porto Alegre, Rio Grande do Sul, Brazil

2 Department of Physical Education, Federal University of Pará, Castanhal, Pará, Brazil

3 Department of Physiotherapy, Santa Catarina State University, Florianópolis, Santa Catarina, Brazil

4 Department of Sports Methods and Techniques, Federal University of Santa Maria, Santa Maria, Brazil

5 Department of Physiotherapy, Federal University of Rio de Janeiro, Rio de Janeiro, Rio de Janeiro, Brazil

\section{Introduction}

Since December 2020, the COVID-19 pandemic has produced more than $170,000,000$ confirmed cases and more than 3,700,000 deaths around the world [1], and Brazil has registered more than $16,900,000$ confirmed cases and more than 470,000 COVID-19-related deaths [1,2]. In an attempt to contain the spread of the disease, numerous countries have adopted social distancing measures, ranging from lockdown to self-isolation [3], reducing the number of relationships and frequency of contact with family, friends, and the community. COVID-19 has particularly affected elderly people with various comorbidities, such as hypertension, diabetes mellitus, cardiovascular and renal disease [4], and chronic 
diseases [5], including Parkinson's disease (PD) [3]. Due to which, people with Parkinson's (PwP) should rigidly follow the social distancing measures $[6,7]$.

The impacts of the COVID-19 pandemic and the consequent social distancing measures changed PwP routine. During self-isolation, factors such as the postponement of non-urgent medical appointments and suspension of complementary therapy further compromised the daily life of PwP $[6,7]$. Thus, the social, economic, and medical consequences of the pandemic have led to lifestyle changes in PwP, reducing overall physical activity levels and increasing mental health disorders $[8,9]$.

Mental health symptoms, such as anxiety and depression, are common non-motor symptoms affecting $30-40 \%$ of PwP population [10-12]. Currently, a limited number of studies have shown the COVID-19 pandemic's toll on mental health $[8,9,13,14]$, and levels of physical activity $[8,9]$ in PwP. Of these, none were conducted in a low-middle-income country.

There is a lack of information about the associations between mental health symptoms and physical activity in PwP during the COVID-19 pandemic. Only one study assessed whether the COVID-19 pandemic was associated with increased psychological distress and decreased physical activity in PD [9], showing that the COVID-19 pandemic leads to a worsening of PD symptoms by evoking psychological distress as well as lifestyle changes, reducing physical activity levels. In the general population, physical activity was associated with depression and anxiety during the COVID-19 pandemic [15]. However, there is a paucity of studies investigating this association between mental health and physical activity levels in PwP in low- and middleincome countries during the COVID-19 pandemic.

To the best of authors' knowledge, no studies investigated the association between mental health and physical activity levels in PwP in low- and middle-income countries during the COVID-19 pandemic. Therefore, the objectives of this study were: (1) to evaluate mental health symptoms in PwP in self-isolation, before and during the COVID-19 pandemic, in Brazil; (2) to explore associations between mental health symptoms and physical activity levels. We hypothesize that PwP in self-isolation in Brazil during the COVID-19 pandemic, are going to experience worsened levels of mental health, and, that lower levels of physical activity are going to be related to an increased probability of mental health symptoms.

\section{Methods}

\section{Study type}

This is an observational, retrospective, cross-sectional survey. PwP were invited to complete an online self-administered and validated questionnaire, after indicating their consent. The COVID-19 pandemic state was officially declared in Brazil on the second week of March 2020. Data collection started 2 months after the pandemic was confirmed. Between 12th and 21st of May 2020, the questionnaire was distributed through PD associations and community groups, University's outreach projects, internet (e-mail) and researchers and University's social media (WhatsApp, Facebook, Messenger, and Instagram). The Ethical Committee of the School of Physical Education, Physical Therapy and Dance at the Federal University of Rio Grande do Sul approved this study and its consent procedure (CAAE 33547920.9.0000.5347).

\section{Sample}

$156 \mathrm{PwP}$ were eligible to participate in the study from the Brazilian territory, who self-report to have clinical diagnosis of PD, both sexes, no age limit, currently residing in Brazil in self-isolation due to COVID-19 pandemic. By self-isolation, we mean those that decided to stay-at-home and only left for essential activities, such as visit health professional, get access to medication and food. All participants included in the study were anonymous and volunteered to participate.

\section{Survey instrument: validity and reliability}

The self-reported levels of physical activity and mental health outcomes of PwP, before and during the COVID-19 pandemic, were obtained using an online questionnaire. A panel was created consisting of 11 experts from the areas of interest [16], with an average of 13 years working with $\mathrm{PD}$, to judge the content validity, construct, clarity and relevance, determining the validity indexes (VI) [17]. The cutoff points were considered for the VI $\geq 0.78$ as a valid instrument [16], and the general average measures of the interclass correlation (ICC) test-retest was 0.99 and Kappa 0.92 [18]. According to the cutoff points, proposed by Cicchetti [19], an agreement higher than 0.75 is considered excellent. Thus, the online questionnaire was also considered reliable. After this process, cognitive interviews were held with a few PwP respondents to check their understanding of the questionnaire and its items [20]. A pilot test was then conducted among 29 participants with PD who answered the questionnaire once, and then again 15 days later to avoid memory interference, and to calculate test-retest reliability.

\section{Variables}

\section{Mental health symptoms}

Mental health symptoms, before and during the pandemic, were assessed by 8 Likert scaled questions, on a 3-point 
scale (ranging from 1 to 3 ; never $=1$, lightly often $=2$, very often $=3$ ), asking the participants if they are feeling anxious, depressive, with fear, and having thoughts of death. In this survey instrument, depression and anxiety were assessed because they are two of the most common mental health symptoms that affect PwP [10-12]. We also assessed fear and thoughts of death as they are psychological stress and emotions that are related to depression and anxiety.

\section{Self-reported physical activity levels}

Self-reported physical activity levels, before and during the pandemic, were assessed by four questions. First, participants were asked: did you practice physical activity before the pandemic? Are you practicing physical activity online during the pandemic? These were dichotomic questions (yes $=1 ;$ no $=2$ ). Then, participants were asked: how you classify your physical activity levels before the pandemic? How you classify your physical activity levels during the pandemic. The response options ranged from 1 to 4 (sedentary $=1$, lightly active $=2$, active $=3$, very active $=4$ ).

\section{Demographic variables}

Demographic data were collected, including sex, age, Brazilian state they lived in, levels of education, and household income. Participants were asked whether they are in selfisolation and to report the number of days in self-isolation. Self-reported previous diagnosis of PD, time of diagnosis, and PD symptoms that bothered most during the period of self-isolation were also evaluated.

\section{Data collection and statistical analysis}

For the statistical analysis, categoric variables are shown as absolute frequencies (\%), and continuous variables as mean (standard deviation) and 95\% confidence intervals.

Data normality was verified using the Shapiro-Wilk test. The Wilcoxon rank-order test was used to evaluate the statistical significance of differences over time (before versus during the pandemic, after 2 months of lockdown) for the following aspects of mental health: anxiety, depression, fear, and thoughts of death. The effect size (d) was determined using the Hodges-Lehmann estimate, and the rank-biserial correlation, interpreted as trivial $<0.1$, small $0.1-0.3$, medium 0.3-0.5, and large $>0.5$.

For the regression analysis, dummy variables were created for the mental health symptoms and physical activity levels, to measure the participants changing over time, based on the following question: did the motor symptoms and physical activity levels get worse from the moment before to during the pandemic? The associations between the mental health symptoms (anxiety, depression, fear and thoughts of death) with self-reported physical activity levels were tested using logistic regression (using dummy variables). First, the models were tested without adjustments (crude), and second, were adjusted for some demographic variables (sex, age, levels of education, PD symptoms, and time of the disease), and physical activity levels (dummy variable). Results from the logistic and linear regression model are presented as the odds ratios (ORs), 95\% confidence intervals and $p$ values. A $5 \%$ significance level was adopted $(p \leq 0.05)$. All tests were performed using the JASP open-source statistical package version 0.11.1 (https://jasp-stats.org/).

\section{Results}

Table 1 presents participants' demographic and general characteristics according to sex. The participants were 156 individuals with PD $(64 \pm 11$ years $)$, women $(n=78$; $63 \pm 11$ years) and men ( $n=78 ; 64 \pm 12$ years), resident in the five Brazilian regions: South $(n=90)$, Southeast $(n=41)$, North $(n=17)$, Northeast $(n=4)$, and Midwest $(n=4)$. According to the participants, $100 \%$ are in self-isolation, $70 \%$ for around 2 months (mean $52 \pm 16$ days). All participants had access to and took PD medication. Regarding socioeconomic data and level of education, most of the population had completed high school (81\%), and received up to 2 minimum wages per month. In relation to the time of diagnosis, $43 \%$ have had PD for less than 5 years, $28 \%$ between 6 and 10 years and 29\% for more than 11 years. Tremor and rigidity were considered the PD symptoms that most bothered the participants during the period of self-isolation.

Figure 1 shows physical activity levels before the pandemic. Before the pandemic, $13 \%$ of the participants reported being sedentary, 38\% lightly active, 39\% active, and $10 \%$ highly active; and $84 \%$ of the participants practiced physical activity, and $16 \%$ not.

\section{Mental health symptoms versus self-reported physical activity levels}

Figure 2 presents a comparison of the results for the mental health symptoms before and during the pandemic. There was a statistical difference over time (before versus during the pandemic), in the mental health symptoms: anxiety [effect size $=-0.52 ; 95 \%$ CI $(-0.70 ;-0.28) ; p<0.001]$, fear [effect size $=-0.58 ; 95 \%$ CI $(-0.76 ;-0.34) ; p<0.001$ ], and thoughts of death [effect size $=-0.43 ; 95 \% \mathrm{CI}(-0.072$; $-0.02) ; p=0.001]$. No statistical differences were found for depression.

A lower physical activity level during the pandemic is related to increase probability of thoughts of death [crude: $\mathrm{OR}=1.84 ; 95 \% \mathrm{CI}(0.98 ; 3.46) ; p=0.05$; adjusted: $\mathrm{OR}=2.98$; $95 \% \mathrm{CI}(-0.01 ; 2.19) ; p=0.05]$. Anxiety, fear and depression 
Table 1 Participants' demographic and general characteristics according to sex

\begin{tabular}{|c|c|c|c|}
\hline Variables & $\begin{array}{l}\text { Total (156) } \\
n(\%) / \text { mean/SD }\end{array}$ & $\begin{array}{l}\text { Women (78) } \\
n(\%) / \text { mean/SD }\end{array}$ & $\begin{array}{l}\text { Men (78) } \\
n(\%) / \text { mean/SD }\end{array}$ \\
\hline Age (years) & $64 \pm 11$ & $63 \pm 11$ & $64 \pm 12$ \\
\hline Self-isolation (days) & $52 \pm 16$ & $53 \pm 15$ & $52 \pm 16$ \\
\hline \multicolumn{4}{|l|}{ Level of education } \\
\hline Elementary school & $30(19)$ & $21(27)$ & $9(11)$ \\
\hline High school & $59(38)$ & $25(32)$ & $34(44)$ \\
\hline University education & $40(26)$ & $20(26)$ & $20(26)$ \\
\hline Postgraduate & $27(17)$ & $12(15)$ & $15(19)$ \\
\hline \multicolumn{4}{|l|}{ Income per month } \\
\hline Up to 2 minimum wages & $68(44)$ & $41(53)$ & $27(35)$ \\
\hline 3 to 4 minimum wages & $42(23)$ & $20(26)$ & $22(28)$ \\
\hline 5 to 10 minimum wages & $34(22)$ & $12(15)$ & $22(28)$ \\
\hline 11 to 20 minimum wages & $8(5)$ & $4(5)$ & $4(5)$ \\
\hline Above 20 minimum wages & $4(3)$ & $1(1)$ & $3(4)$ \\
\hline \multicolumn{4}{|l|}{ Time of diagnosis } \\
\hline Less than 5 years & $67(43)$ & $33(42)$ & $34(43)$ \\
\hline $6-10$ years & $44(28)$ & $20(26)$ & $24(31)$ \\
\hline More than 11 years & $45(29)$ & $25(32)$ & $20(26)$ \\
\hline \multicolumn{4}{|l|}{ Symptom that most bothered } \\
\hline Tremor & $44(28)$ & $23(30)$ & $21(27)$ \\
\hline Rigidity & $46(30)$ & $27(35)$ & $19(24)$ \\
\hline Bradykinesia & $23(15)$ & $6(8)$ & $17(22)$ \\
\hline Gait problems & $36(23)$ & $19(24)$ & $17(22)$ \\
\hline Freezing & $7(4)$ & $3(4)$ & $4(5)$ \\
\hline
\end{tabular}

Note: $S D$ standard deviation, $\%$ percentage
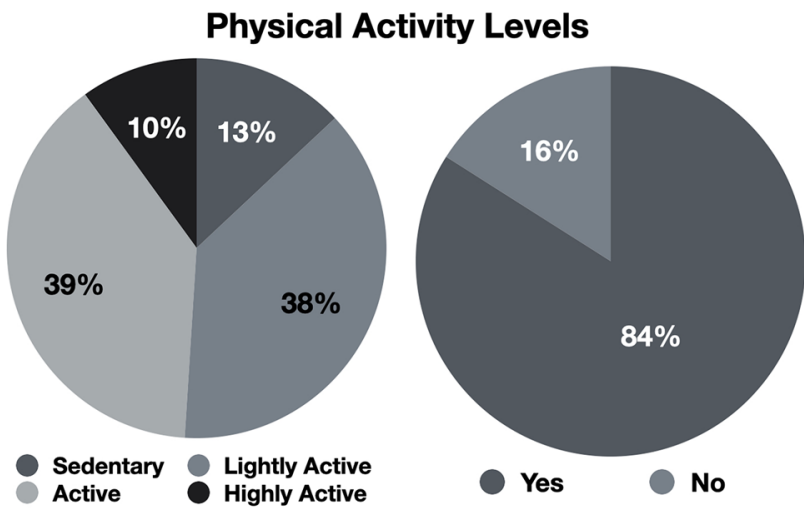

Fig. 1 Case frequencies (\%) of physical activity levels before the COVID-19 pandemic

were not associated with physical activity levels. The results of the logistic regression models are presented in Table 2.

\section{Discussion}

The study findings showed the participants were more anxious, depressed, fearful, and more frequently thought about death during the pandemic. Lower levels physical activity during the pandemic are related to increased probability of thoughts of death, confirming our hypothesis.

The results of this study indicated that the COVID-19 pandemic together with the self-isolation measures affected fear, thoughts of death, and anxiety, when comparing before and during the pandemic. Our findings are consistent with previous studies $[3,8,9,13,14]$, which also indicated that PwP experience heightened levels of anxiety during the COVID-19 pandemic, worsening the pre-existent nonmotor symptoms [10-12]. In PwP, the feelings of fear can be related with the perception of greater risk of contracting the COVID-19 virus, due to a subjunctive chronical medical condition [13], and was most often attributed to anxiety [21]. During the pandemic, the fear increased anxiety in healthy people and intensified the symptoms of individuals that already presented psychiatric disorders [22]. In addition, studies carried out in Japan [22], China [23], Brazil [24], and United Kingdom [25] found a high prevalence of anxiety symptoms in the general population during the COVID-19 

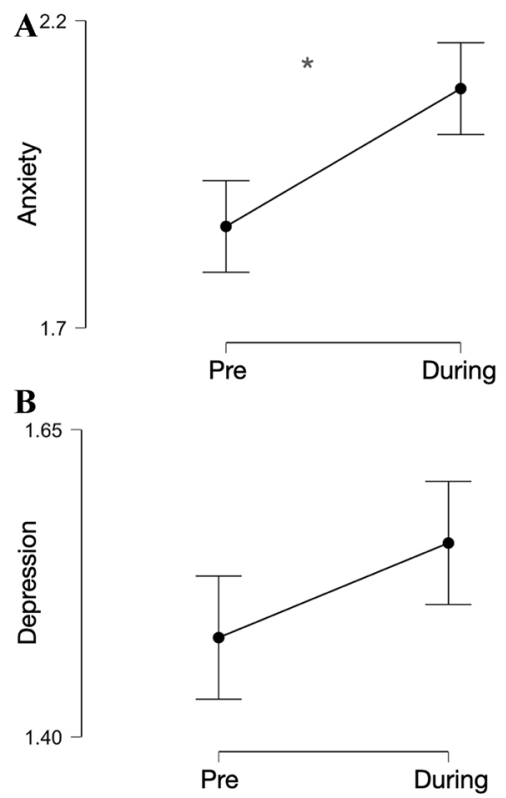

C

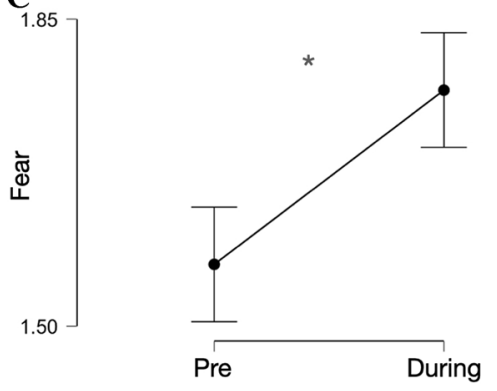

D

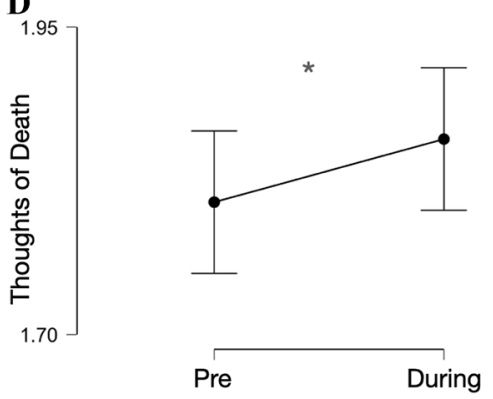

Fig. 2 Comparison of mental health symptoms, before and during the pandemic, in PwP. Note: $*$ represents differences between before and during the pandemic at $p<0.05$

pandemic, suggesting that the pandemic, together with lockdown and self-isolation measures are impacting the mental health of the general population. Therefore, to attenuate this situation, health workers must encourage communication between PwP and their families, caregivers, and friends to minimize the effects of social distancing and self-solation [26].

In addition, during the pandemic, lower physical activity levels are related to increased probability of thoughts of death. These results were in line with a previous study that also pointed out that reduced levels of physical activity are correlated with worsening symptoms in PwP during the COVID-19 pandemic [9], and also with Wolf et al. [15] that found that performing physical activity during COVID-19 is associated with less depression and anxiety.

The World Health Organization, as part of its public health response, has worked to develop a set of new materials on the mental health during the COVID-19 pandemic, to support mental and psychological well-being in different target groups [27, 28]. Highlighting the chronic and progressive characteristics of $\mathrm{PD}$, strategies to promote health for PwP must be adapted to the new reality to not further compromise mental health problems, and increase levels of physical activity. Digital technology and telehealth have become resources for providing care and assistance for PwP. Telemedicine, telerehabilitation and the provision of online physical activity are alternatives suggested in the literature to reduce the impact of social isolation, and mitigate the worsening of PD non-motor symptoms [6, 14, 15, 29, 30].

The results of this study are relevant and novel. To the authors knowledge, this is the first study in this topic in Brazil, which investigated the effects due of the COVID-19 pandemic and the social isolation measures on mental health outcomes in PwP by comparing the before and during pandemic periods, and to explore associations between mental health symptoms and physical activity levels.

However, the study has some limitations. First, the small number of responses obtained from the Midwest and Northeast Brazilian regions. Second, all data were self-reported potentially suffering from social desirability bias. In addition, pre-pandemic variables were assessed retrospectively and could suffer from recall bias. Prospective longitudinal studies are required to verify the long-term effects of the COVID-19 pandemic on PwP mental health and levels of physical activity.

\section{Conclusion}

Self-isolation during the pandemic has increased anxiety, fear and thoughts of death among people with PD in comparison with the period before COVID-19. Time of diagnosis and PD symptoms presented no relationship with any of the analyzed mental health symptoms.

A lower physical activity level during the pandemic was related to an increased probability of thoughts of death. Anxiety, depression and fear presented no relationship with lower physical activity levels.

Online-based health promotion strategies should be proposed to mitigate mental health problems among PwP during the COVID-19 pandemic. 
Table 2 Logistic regression analysis determining risk factors for mental health symptoms according to physical activity levels

\begin{tabular}{|c|c|c|c|c|c|c|c|c|}
\hline & \multicolumn{4}{|c|}{ Crude } & \multicolumn{4}{|c|}{ Adjusted } \\
\hline & OR & $95 \% \mathrm{CI}$ & & $p$ & OR & $95 \% \mathrm{CI}$ & & $p$ \\
\hline \multicolumn{9}{|l|}{ Anxiety } \\
\hline PA level & 0.90 & 0.46 & 1.76 & 0.75 & 1.64 & -0.21 & 1.20 & 0.17 \\
\hline \multicolumn{9}{|l|}{ Depression } \\
\hline PA level & 1.00 & 0.46 & 2.17 & 0.99 & 2.37 & -0.061 & 1.79 & 0.07 \\
\hline \multicolumn{9}{|l|}{ Fear } \\
\hline PA level & 0.76 & 0.37 & 1.55 & 0.46 & 1.40 & -0.41 & 1.08 & 0.37 \\
\hline \multicolumn{9}{|c|}{ Thoughts of death } \\
\hline PA level & 1.84 & 0.98 & 3.46 & $0.05^{*}$ & 2.98 & -0.01 & 2.19 & $0.05^{*}$ \\
\hline
\end{tabular}

Note: $P A$ Physical activity, $C I$ confidence interval, $O R$ Odds Ratio, ${ }^{*} p \leq 0.05=$ statistical significance. The models presented are: crude, no adjustments; adjusted for sex, age, levels of education, PD symptoms that bothered most during the period of self-isolation, time of the disease, and self-reported physical activity level
Supplementary Information The online version contains supplementary material available at https://doi.org/10.1007/s11332-021-00868-y.

\section{Acknowledgements Not applicable.}

Funding The authors received no specific funding for this work.

Data availability All relevant data are within the manuscript, in figures, tables and in supporting information file.

Code availability Not applicable.

\section{Declarations}

Conflict of interest The authors have no conflict of interest to declare.

Ethical approval The Ethical Committee of School of Physical Education, Physical Therapy and Dance of the Federal University of Rio Grande do Sul approved this work (CAAE 33547920.9.0000.5347). All procedures performed in the study were in accordance with the 1964 Helsinki declaration.

Consent to participate All participants read and signed the informed consent.

Consent for publication Not applicable.

\section{References}

1. World Health Organization (WHO). Coronavirus disease (COVID-19) data. [Cited 8 June 2021]. Available from: https:// www.who.int/data\#reports

2. Ministry of health. Panel of cases of coronavirus disease 2019 (COVID-19) in Brazil by the Ministry of Health. 2020 Oct 19 [Cited 8 June 2021]. Available from: https://covid.saude.gov.br/

3. Prasad S, Holla VV, Neeraja K, Surisetti BK, Kamble N, Yadav R et al (2020) Parkinson's disease and COVID-19: perceptions and implications in patients and caregivers. Mov Disord 35(6):912914. https://doi.org/10.1002/mds.28088
4. Chen T, Wu D, Chen H, Yan W, Yang D, Chen G et al (2020) Clinical characteristics of 113 deceased patients with coronavirus disease 2019: retrospective study. BMJ 368:m1091. https:// doi.org/10.1136/bmj.m1091

5. Haybar H, Kazemnia K, Rahim F (2020) Underlying chronic disease and COVID-19 infection: a state-of-the-art review. Jundishapur J Chronic Dis Care 9(2):e103452. https://doi.org/ 10.5812/jjcdc. 103452

6. Papa SM, Brundin P, Fung VS, Kang UJ, Burn DJ, Colosimo C et al (2020) Impact of the COVID-19 pandemic on Parkinson's disease and movement disorders. Mov Disord 35(5):711-715. https://doi.org/10.1002/mds. 28067

7. Helmich RC, Bloem BR (2020) The impact of the COVID-19 pandemic on Parkinson's disease: hidden sorrows and emerging opportunities. J Parkinsons Dis 10(2):351-354. https://doi.org/ 10.3233/JPD-202038

8. Balci B, Aktar B, Buran S, Tas M, Donmez Colakoglu B (2021) Impact of the COVID-19 pandemic on physical activity, anxiety, and depression in patients with Parkinson's disease. Int J Reahbil Res 44(2):173-176. https://doi.org/10.1097/MRR.00000 00000000460

9. Van der Heide A, Meinders MJ, Bloem BR, Helmich RC (2020) The impact of the COVID-19 pandemic on psychological distress, physical activity, and symptom severity in Parkinson's disease. J Parkinson's Dis 10(4):1355-1364. https://doi.org/10. 3233/JPD-202251

10. Timmer MHM, van Beek MHCT, Bloem BR, Esselink RAJ (2017) What a neurologist should know about depression in Parkinson's disease. Pract Neurol 17(5):359-368. https://doi. org/10.1136/practneurol-2017-001650

11. Broen MP, Narayen NE, Kuijf ML, Dissanayaka NN, Leentjens AF (2016) Prevalence of anxiety in Parkinson's disease: a systematic review and meta-analysis. Mov Disord 31(8):11251133. https://doi.org/10.1002/mds. 26643

12. Chuquilín-Arista F, Álvarez-Avellón T, Menéndez-González M (2020) Prevalence of depression and anxiety in Parkinson disease and impact on quality of life: a community-based study in Spain. J Geriatr Psychiatry Neurol 33(4):207-213. https:// doi.org/10.1177/0891988719874130

13. Salari M, Zali A, Ashrafi F, Etemadifar M, Sharma S, Hajizadeh $\mathrm{N}$ et al (2020) Incidence of anxiety in Parkinson's disease during the coronavirus disease (COVID-19) pandemic. Mov Disord 35(7):1095-1096. https://doi.org/10.1002/mds.28116 
14. Shalash A, Roushdy T, Essam M, Fathy M, Dawood NL, Abushady EM et al (2020) Mental health, physical activity, and quality of life in Parkinson's disease during COVID-19 pandemic. Mov Disord 35(7):1097-1099. https://doi.org/10.1002/mds.28134

15. Wolf S, Seiffer B, Zeibig JM, Welkerling J, Brokmeier L, Atrott B, Ehring T, Schuch FB (2021) Is physical activity associated with less depression and anxiety during the COVID-19 pandemica rapid systematic review. Sports Med. https://doi.org/10.1007/ s40279-021-01468-z

16. Halek M, Holle D, Bartholomeyczik S (2017) Development and evaluation of the content validity, practicability and feasibility of the Innovative dementia-oriented assessment system for challenging behaviour in residents with dementia. BMC Health Serv Res 17(554):1-26. https://doi.org/10.1186/s12913-017-2469-8

17. de Sá-Caputo DC, Sonza A, Bachur JA, Bernardo-Filho M (2020) Development, validation and reliability of a questionnaire to evaluate the changes on the level of physical exercises and in daily life habits due to COVID-19 pandemic social distancing. Acta Biomed. https://doi.org/10.23750/abm.v91i3.9888

18. Portney LG, Watkins MP (2015) Foundations of clinical research: applications to practice, 3rd edn. Davies Company, Philadelphia

19. Cicchetti DV (1994) Guidelines, criteria, and rules of thumb for evaluating normed and standardized assessment instruments in psychology. Psychol Assess 6(4):284-290. https://doi.org/10. 1037/1040-3590.6.4.284

20. Artino AR, La Rochelle JS, Dezee KJ, Gehlbach H (2014) Developing questionnaires for educational research: AMEE guide No. 87. Med Teach 36(6):463-474. https://doi.org/10.3109/0142159X. 2014.889814

21. Feeney MP, Xu Y, Surface M et al (2021) The impact of COVID19 and social distancing on people with Parkinson's disease: a survey study. NPJ Parkinsons Dis 7(10):1-10. https://doi.org/10. 1038/s41531-020-00153-8

22. Shigemura J, Ursano RJ, Morganstein JC, Kurosawa M, Benedek DM (2020) Public responses to the novel 2019 coronavirus (2019$\mathrm{nCoV}$ ) in Japan: mental health consequences and target populations. Psychiatry Clin Neurosci 74(4):281-282. https://doi.org/10. 1111/pcn.12988

23. Wang C, Pan R, Wan X, Tan Y, Xu L, Ho CS et al (2020) Immediate psychological responses and associated factors during the initial stage of the 2019 coronavirus disease (COVID-19) epidemic among the general population in China. Int J Environ Res Public Health 17(5):1-25. https://doi.org/10.3390/ijerph17051729

24. Schuch FB, Rugero AB, Jacob M, Davy V, Joseph FBS, Igor G et al (2020) Associations of moderate to vigorous physical activity and sedentary behavior with depressive and anxiety symptoms in self-isolating people during the COVID-19 pandemic: a crosssectional survey in Brazil. Psychiatry Res 292:113339. https:// doi.org/10.1016/j.psychres.2020.113339

25. Smith L, Jacob L, Yakkundi A, McDermott D, Armstrong NC, Barnett Y et al (2020) Correlates of symptoms of anxiety and depression and mental wellbeing associated with COVID-19: a cross- sectional study of UK-based respondents. Psychiatry Res. https://doi.org/10.1016/j.psychres.2020.113138

26. Paz TSR, Macedo ARS, Silva AEL, Pessoa G, Martins NIM, Miranda HL et al (2020) Challenges in treating people with Parkinson's disease during the COVID-19 pandemic. Bull Fac Phys Ther 25(17):1-3. https://doi.org/10.1186/s43161-020-00017-5

27. World Health Organization (WHO). Mental health and COVID19. 2020 May 29 [Cited 10 Oct 2020]. Available from: https:// www.euro.who.int/en/health-topics/noncommunicable-diseases/ mental-health/data-and-resources/mental-health-and-covid-19

28. World Health Organization (WHO). Mental health and psychosocial considerations during the COVID-19 outbreak. 2020 [Cited 10 Oct 2020]. Available from: https://www.who.int/docs/defau lt-source/coronaviruse/mental-health-considerations.pdf?sfvrsn= 6d3578af 10

29. Stoessl JA, Kailash PB, Merello M (2020) Movement disorders in the world of COVID-19. Mov Disord 35(5):709-710. https:// doi.org/10.1002/mds. 28069

30. Langer A, Gassner L, Flotz A, Hasenauer S, Gruber J, Wizany L, Pokan R, Maetzler W, Zach H (2021) How COVID-19 will boost remote exercise-based treatment in Parkinson's disease: a narrative review. NPJ Parkinsons Dis 7(25):1-9. https://doi.org/ 10.1038/s41531-021-00160-3

Publisher's Note Springer Nature remains neutral with regard to jurisdictional claims in published maps and institutional affiliations. 